\title{
Investigating Mutations to Reduce Huntingtin Aggregation by Increasing Htt-N-Terminal Stability and Weakening Interactions with PolyQ Domain
}

\author{
Mohamed R. Smaoui, ${ }^{1,2}$ Cody Mazza-Anthony, ${ }^{3}$ and Jérôme Waldispühl ${ }^{3}$ \\ ${ }^{1}$ Division of Experimental Medicine, Faculty of Medicine, McGill University, Montreal, QC, Canada \\ ${ }^{2}$ Institut de Recherches Cliniques de Montreal (IRCM), Montreal, QC, Canada \\ ${ }^{3}$ School of Computer Science, McGill University, Montreal, QC, Canada \\ Correspondence should be addressed to Mohamed R.Smaoui; mohamed.smaoui@mail.mcgill.ca
}

Received 28 September 2016; Revised 14 November 2016; Accepted 16 November 2016

Academic Editor: Prem Chapagain

Copyright ( 2016 Mohamed R. Smaoui et al. This is an open access article distributed under the Creative Commons Attribution License, which permits unrestricted use, distribution, and reproduction in any medium, provided the original work is properly cited.

\begin{abstract}
Huntington's disease is a fatal autosomal genetic disorder characterized by an expanded glutamine-coding CAG repeat sequence in the huntingtin (Htt) exon 1 gene. The Htt protein associated with the disease misfolds into toxic oligomers and aggregate fibril structures. Competing models for the misfolding and aggregation phenomena have suggested the role of the Htt-N-terminal region and the CAG trinucleotide repeats (polyQ domain) in affecting aggregation propensities and misfolding. In particular, one model suggests a correlation between structural stability and the emergence of toxic oligomers, whereas a second model proposes that molecular interactions with the extended polyQ domain increase aggregation propensity. In this paper, we computationally explore the potential to reduce Htt aggregation by addressing the aggregation causes outlined in both models. We investigate the mutation landscape of the Htt-N-terminal region and explore amino acid residue mutations that affect its structural stability and hydrophobic interactions with the polyQ domain. Out of the millions of 3-point mutation combinations that we explored, the (L4K E12K K15E) was the most promising mutation combination that addressed aggregation causes in both models. The mutant structure exhibited extreme alpha-helical stability, low amyloidogenicity potential, a hydrophobic residue replacement, and removal of a solvent-inaccessible intermolecular side chain that assists oligomerization.
\end{abstract}

\section{Background}

Huntington Disease (HD) is a fatal autosomal dominant genetic disorder that is characterized by CAG trinucleotide repeats in the huntingtin (Htt) exon 1 gene $[1,2]$. HD is part of the amyloidoses group of diseases characterized by large deposits of amyloid proteins that inflame and weaken cells, leading to their destruction. Parkinson's, Alzheimer's, and diabetes, to name a few, also belong to the same amyloidosis group and have been shown to be greatly aggravated by amyloids [3-5]. These diseases caused or implicated by protein misfolding are usually categorized by the accumulation of insoluble amyloid proteins that form into long fibrils in the body [6]. The build-up of these fibrils causes tissue degradation and appears at the onset of the particular disease. In HD, both the alpha-helical Htt-N-terminal region and the
CAG trinucleotide repeats (polyQ domain) are believed to participate in the misfolding process of the huntingtin protein into beta-sheet rich amyloids that aggregate into potentially toxic oligomeric species and fibril structures [7-9]. The wildtype form of $\mathrm{Htt}$ is 3144 amino acids in length; however the amyloid form has more CAG repeats causing an extended polyQ domain. The polyQ domain begins at residue 18, as drawn in Figure 1. The rate of aggregation greatly depends on the flanking regions of the polyQ domain. In some cases, the $\mathrm{N}$-terminal domain has been shown to adopt an alpha-helical structure that affects aggregate formation [8] and mutations have been observed to influence fibril formation [10-15].

Htt is found everywhere in human tissues but is significantly expressed in the cerebral cortex of the brain. The homology of Htt is not well known; hence identifying 


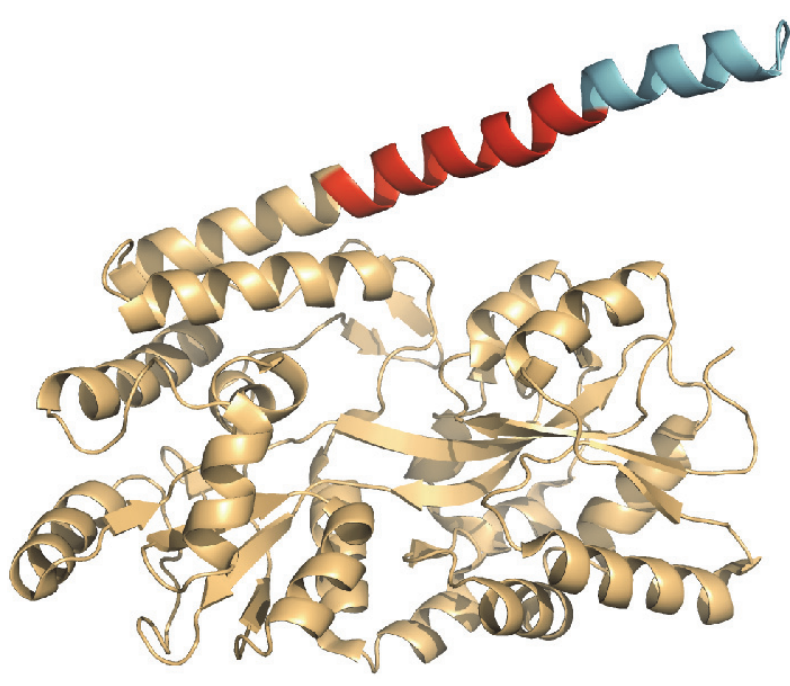

FIgURE 1: Chain B from PDB 3IO6. Orange residues are positions 1370 and part of the Htt amino terminal region. The red positions 371387 are the 17 residues of the Htt-N-terminal region implicated in the formation of amyloids (MATLEKLMKAFESLKSF). The blue residues are GLN repeats attached to the $\mathrm{N}$-terminal region.

the exact function of the protein has been very difficult. Moreover, the Htt protein interacts with more than 100 different proteins in the body, displaying numerous functions [16]. The ubiquitous Htt in the human body is involved in microtubule machinery and vesicle trafficking $[17,18]$. The presence of Htt in the brain of mice has been pivotal to proper development due to its maintenance of ER in neurons [19]. The exact trigger to commence the oligomers and fibril formation is not well defined. Nevertheless, the onset of symptoms is usually attributed to a toxic gain of function by the mutant amyloid [20-22]. This gain of function usually enables a pathway that destabilizes the Htt-N-term to start forming beta-sheets.

There is no known cure for HD and approximately 30,000 people possess the gene that causes HD in the US and Canada. The prevalence of the disease is mainly among Caucasians in a ratio of 5-7 per 100,000 people [23]. As the disease progresses it causes neuronal damage to the brain causing a variety of symptoms including involuntary movements, decreased cognitive function, and psychotic behavior [24]. Current treatments for the disease remain mostly ineffective due to the lack of knowledge surrounding the function of the huntingtin protein [25]. The disease is characterized by intracellular inclusions that form in the GABA-ergic neurons in the brain leading to cell death [26]. The death of these essential neurons leads to uncoordinated movements, erratic body movements, and changes in behavior [27]. Degradation of neuronal pathways leads to dementia and loss of bodily control resulting in injury and eventually full-time medical care. In its last stages, the disease can cause death by triggering failure of most of the body's vital systems.

Stem cell therapy has recently been shown to be a potential treatment for damaged brain cells, allowing for the reversibility of the damages caused by the disease [28]. The disease begins in the central nervous system as large amounts of the healthy form of the Htt protein (native form) misfold and become toxic. The transition between a native and amyloid structure is governed by an amyloidogenic energy barrier that is easier to surpass when the Htt protein is unstable. Hence theoretically, increasing the stability of the Htt protein is one way to potentially decrease the emergence of amyloids and allow for a slower progression of the disease.

Currently, there are two prominent aggregation models outlining how the Htt protein misfolds and aggregates. The first model suggests that the Htt-N-terminal region is the key player behind aggregation, existing normally in a random coil conformation and folding into a helical structure upon interaction with a second Htt-N-terminal to form an oligomer structure [29]. The Htt-N-terminal region is present in helical form in fibrils of huntingtin exon 1, suggesting an involvement in fibril formation, possibly via alpha-helical interactions that create oligomers $[8,30-$ 32]. The Lysine residues (6 and 15) in the Htt-N-terminal region are believed to form an intermolecular side chain interaction and are solvent-inaccessible in the aggregate state [15]. Mutating any of these residues could potentially weaken or break oligomerization. More recently, Arndt et al. strongly confirmed the high degree of helical propensity of the Htt-Nterminal region in solution and observed that some Htt-Nterminal regions turn back on themselves and self-associate with helical bundles [9]. The second model suggests that aggregation of the huntingtin protein occurs as a result of the interaction between the Htt-N-terminal region and huntingtin's extended polyQ domain (the domain cross-talk model) $[33,34]$. This model proposes that the hydrophobic residues of the Htt-N-terminal region interact with the polyQ domain and stabilize its structure, driving fibrillization and bypassing the oligomer state.

Computational models have served to increase our understanding of biological processes throughout time. The problem of multiple protein sequence alignment [35], the mapping of molecular evolution [36, 37], and modeling the dynamics of molecules could have only been tackled with the use of computational power [38]. Computational methods assisted analysis on genomic data, mapping of nucleotide and amino acid sequence relationships, exploring protein domains and structures, and storing data sets $[39,40]$. The development of computationally intensive techniques and algorithms improved efficient access and use of biological data leading to immediate growth in the fields of drug design, drug discovery, gene finding, and protein structure prediction. The aim of this article is to computationally explore the potential to reduce Htt aggregation by simultaneously addressing the aggregation causes outlined in both models. We investigate the mutation landscape of the Htt$\mathrm{N}$-terminal region and design potential amino acid residue mutations that can be applied to lower the amyloidogenicity of Htt. We do not promote one aggregation model over the other; rather, we explore mutations that consolidate both aggregation models. To perform this, we outline three procedures. First, we explore the entire stability landscape of the $\mathrm{Htt}-\mathrm{N}$-terminal region and identify mutation combinations that significantly increase helical stability to prevent selfassociation of the Htt-N-terminal region into helical bundles 


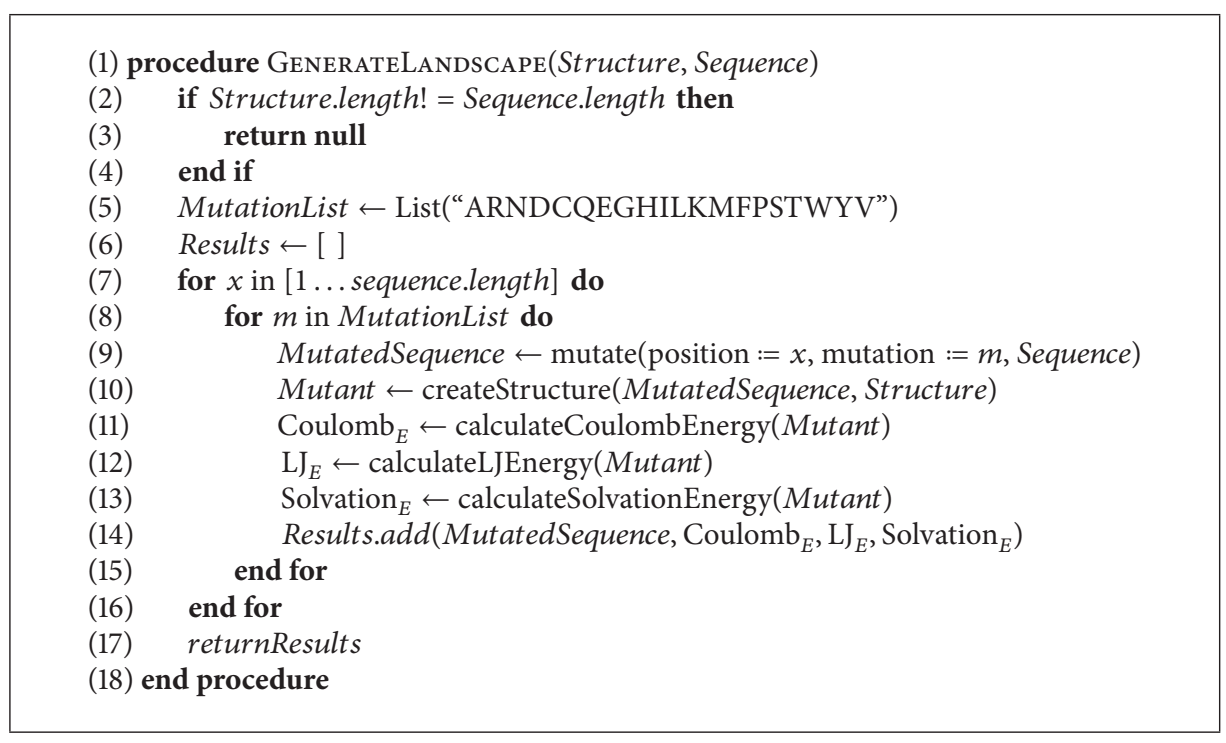

Algorithm 1: Generating the mutational landscapes of the Htt-N-term.

and lower the aggregation propensity suggested by the first model. Second, we analyze the most promising mutation combinations from the previous step in search for mutations that replace hydrophobic residues with charged amino acids to weaken interactions with the polyQ domain outlined in the second model. Finally, we explore any mutation combinations that additionally break the intermolecular side chain between residues 6 and 15, further lowering oligomerization potential. Together, all three procedures simultaneously address the theories behind $\mathrm{Htt}$ aggregation proposed by both models. Our results indicate that the mutation combination (L4K E12K K15E) is the most promising mutation that satisfies all three conditions. We confirm with molecular dynamics productions that this mutation combination does not alter native form and results in an Htt- $\mathrm{N}$-terminal mutant structure that exhibits extreme stability and potentially lowers amyloidogenicity. Although not a substitute for experimental studies, computational simulations of proteins can provide insight and test procedures that remain difficult to study experimentally.

\section{Materials and Methods}

In this section, we outline the technical procedure we used to map the mutation landscape of the $\mathrm{Htt}-\mathrm{N}$-terminal region and explain in detail how we assess the stability of residues on the protein fragment structure. We performed a bruteforce procedure to calculate the stability effect of every possible single-point mutation in the 17 residues of the Htt$\mathrm{N}$-terminal region and used the results to efficiently estimate the effect of 3-point mutations on the protein fragment.

2.1. Exploring Single-Point Mutation Landscape. Algorithm 1 is inspired from Smaoui and Waldispühl [41] and outlines the detailed procedure we used to generate the mutational landscape of the Htt-N-term. Starting with the helical Htt$\mathrm{N}$-term PDB structure in native form (PDB 3IO6, residues
371-387; see Figure 1), we mutated every residue on the structure backbone into all the other possible 19 canonical amino acids. For each single-point mutation experiment, we compute the Lennard-Jones (LJ), Coulomb, and solvation energy terms. While the LJ and Coulomb measure the electrostatic potential and charges between atoms and solvation measures the interactions with a water surface, together, the sum of the three terms provides a good estimate for the stability of a protein structure, as given by the following:

$$
E=\text { Solvation }+\mathrm{LJ}+\text { Coulomb. }
$$

Low values indicate stable energetics, while high energy values suggest unfavourable destabilizing interactions. The single-point mutation results generate the $3 \mathrm{D}$ landscape plot in Figure 2. The amino acid length of the Htt-N-term makes up 1 dimension, the 20 possible amino acid mutations make up the second dimension, and the energy values produced by a (residue, mutation) pair make up the third dimension.

The LJ and Coulomb terms were calculated using the standard definitions given by the GROMACS 4.5 [42] molecular dynamics package using the GROMOS96 53a6 [43] force field along with the SPC [44] water model. We used a cutoff of $10 \AA$ for van der Waals and short range electrostatic interactions and calculated long range electrostatic interactions using a particle mesh Ewald sum $[45,46]$.

For every mutation, the sum of LJ, Coulomb, and solvation energies is computed and compared to the nonmutant structure energies as shown in the following:

$$
\Delta E=E-E_{0},
$$

where $E_{0}$ is the energy of the native nonmutant Htt-N-term and $E$ is the energy of a native mutant structure.

The $\Delta E$ value measures the difference in energy caused by introducing a mutation to the Htt-N-term structure. Positive $\Delta E$ values correlate to a decrease in stability and negative $\Delta E$ values correlate to an increase in stability. 


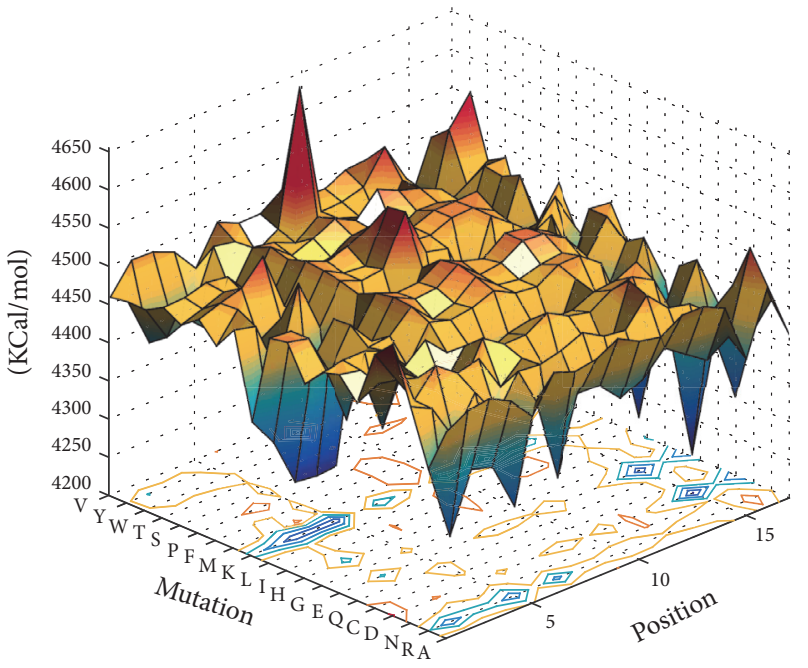

(a) Htt-N-terminal mutation landscape

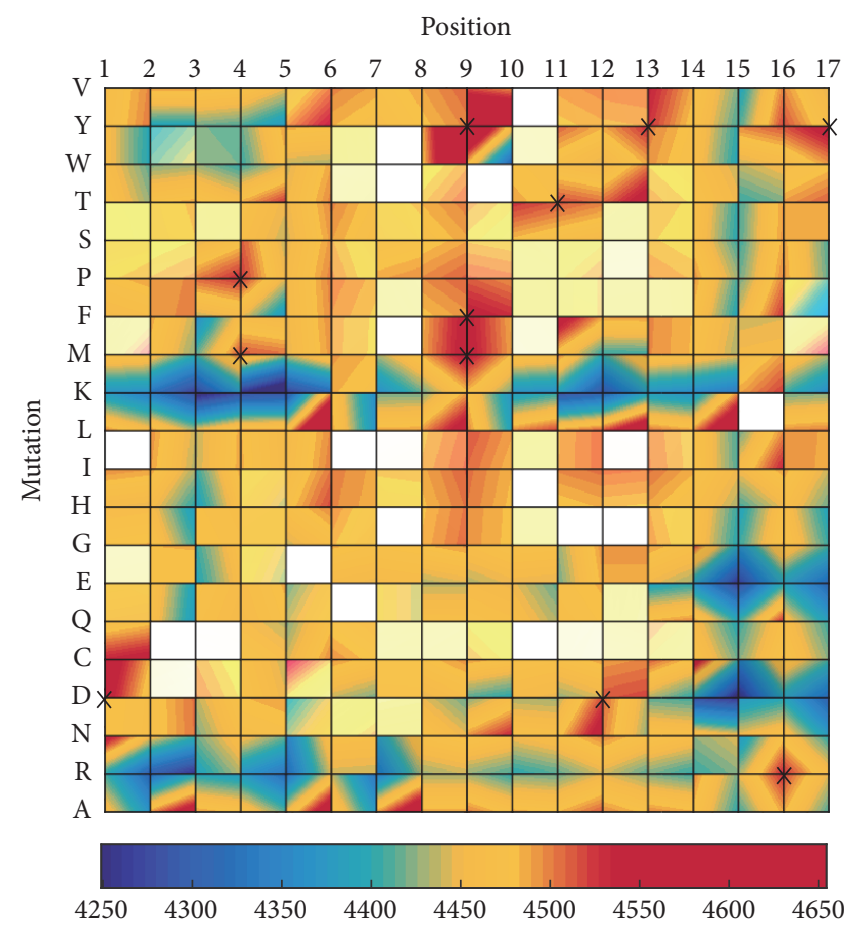

(b) $2 \mathrm{D}$ projection of the landscape

Figure 2: Single-point mutation landscape of the Htt-N-terminal region. Stable mutants have low energies (blue), while destabilizing mutations have the highest energies (red). Energies are in $\mathrm{KCal} / \mathrm{mol}$.

2.2. Solvation Energy. The solvation energy term used to calculate the interactions of our mutant structures with a water surface was computed by AquaSol $[47,48]$ and solves the following:

Solvation

$$
\begin{aligned}
= & F_{\left(p_{0}, C_{\mathrm{dip}}\right)}-F_{(0,0)} \\
& -\left(k_{\mathrm{B}} T \frac{\ln \left(1-N_{A} C_{\mathrm{dip}} a^{3}\right)}{N_{A} C_{\mathrm{dip}} a^{3}}\right) \int_{\text {solvent }} d \mathbf{r} \rho_{\mathrm{dip}}(\mathbf{r}),
\end{aligned}
$$

where $F_{\left(p_{0}, C_{\text {dip }}\right)}$ defines the free energy of a protein system defined at dipoles of moment values $p_{0}$ and concentration $C_{\text {dip }}, F_{(0,0)}$ is the free energy of a protein system with solvent concentration set to zero, $a^{3}$ is the lattice grid size volume of the solvent, $k_{\mathrm{B}}$ is the Boltzmann constant, $T$ is the temperature in Kelvin, and $\mathbf{r}$ is the surface definition, solventaccessible surface probe.

The solvation energy is calculated during formation of the protein when it is in water. The tool utilizes the hydrogen bonds between molecules, the $\mathrm{pH}$, and the temperature to efficiently solve the dipolar nonlinear Poisson-BoltzmannLangevin equation using a fast and detailed dipolar water model. We used AquaSol with the following setup: atomic charges and radii assigned with PDB2PQR using CHARMM force field at neutral $\mathrm{pH}$, a grid of $257 \times 257$ points spaced by $1 \AA$, a temperature of $300 \mathrm{~K}$, and a solvent-accessible surface with an Rprobe of $1.4 \AA$. All hydrogen bonds were optimized. We used a trilinear interpolation protocol for projection of fixed charges on the grid, a lattice grid size for the solvent: $a$ $=2.8 \AA$, and solvent made of dipoles of moment $p_{0}=3.00 \mathrm{D}$ at a concentration of $C_{\text {dip }}=55 \mathrm{M}$. No salt was added to the solution and small ions $\left(\mathrm{Na}^{+}, \mathrm{Cl}^{-}\right)$were used to equilibrate the system when needed. The electrostatic potential was set to zero at the boundaries, and the stopping criteria for residual were sent to $1 \cdot 10^{-6}$ (when possible).

2.3. Performing Mutations. We mutated every residue in the Htt-N-term region into the other 19 canonical amino acids using SCWRL4 [49], a tool that computes the van der Waals forces and hydrogen bond interactions to determine the electron densities of different areas of the protein and predict the side chain conformations to a backbone structure. For every mutation, SCWRL4 fit the new mutant amino acid sequence onto the original Htt-N-terminal backbone to produce a mutant Protein Data Bank (PDB) structure.

2.4. Molecular Dynamics Productions. Subsequent to generating the mutant PDB structures for the Htt-N-term, we perform short energy minimization (EM) runs to relax the structures and remove any steric clashes that were introduced by SCWRL4. The difference of the sums of the LJ, Coulomb, and solvation energy terms (2) between the mutants and nonmutant structures was used to rank the stability of the mutations. Mutants that have a $\Delta E$ that is negative are more stable than the nonmutant structure. Structures that were deemed very stable (low $\Delta E$ ) were then prepared for molecular dynamics (MD) production to formally computationally test structure stability over time. 


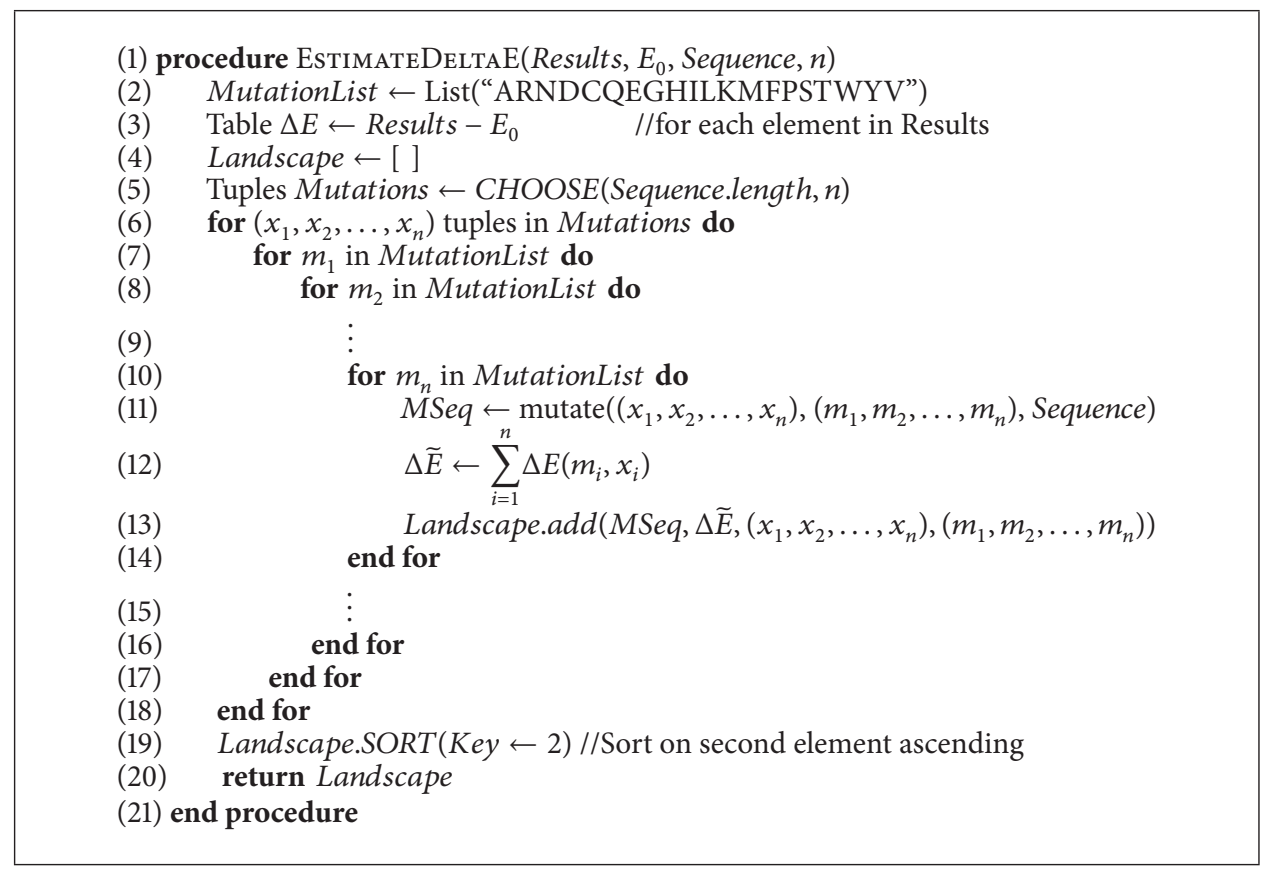

Algorithm 2: Generating $\Delta \widetilde{E}$ for $n$-point mutations.

We used the GROMACS 4.5 [42] molecular simulation package to run molecular dynamics (MD) and energy minimization simulations. Our mutant molecules were solvated in a cubic box (with a minimum distance of $35 \AA$ from any edge of the box to any atom) and neutralized with chloride ions and modeled using the GROMOS96 53a6 force field along with the SPC water model. We used a cutoff of $10 \AA$ for van der Waals and short range electrostatic interactions and calculated long range electrostatic interactions using a particle mesh Ewald sum $[45,46]$. Simulations were prepared for a full MD run in both isothermal-isobaric (100 ps) and canonical equilibration (100 ps) ensembles. Temperature and pressure were controlled at $300 \mathrm{~K}$ and 1 bar using the velocity rescaling thermostat and the Parrinello-Rahman barostat, respectively. A linear constraint solver was used to keep all bonds at their equilibrium length. Approximately twenty-five million time steps were used with an integration time step of $2 \mathrm{fs}$ to assess any potential turbulence introduced into the molecules by mutations. The system's coordinates were saved every $10 \mathrm{ps}$ for further analysis.

2.5. Assessing Structural Deviations. Following EM relaxation, we use MD to compute the stability of the mutant structure over time by analyzing amino acid perturbations using RMSD and RMSF plots. An RMSD plot measures the root mean-square deviations, in angstroms, of the $C_{\alpha}$ atom positions in protein's residues over a simulation run, whereas the RMSF measures the root mean-square fluctuations, a measure of the deviation between the position of a particle $i$ over a simulation run given by

$$
\mathrm{RMSF}=\frac{1}{T} \sum_{t_{j}=1}^{T}\left(x_{i}\left(t_{j}\right)-\tilde{x}_{i}\right)^{2},
$$

where $T$ is the total simulation time and $\widetilde{x}_{i}$ is the reference position of particle $i$. Low RMSF at a particular mutation site suggests the absence of local residual instability.

2.6. Estimates for Multiple-Point Mutations. The problem of calculating $n$-point mutations (multiple simultaneous singlepoint mutations) grows exponentially in time and space as $n$ increases in size. Even for a reasonable $n=3$, the number of structures that we need to consider grows to the millions. To circumvent these calculations, we use the data from singlepoint mutations to estimate the effect of 3-point mutations. This method has been shown to return accurate estimates [41]. We outline the procedure of calculating an approximate $\Delta E$ for multiple-point mutations in Algorithm 2. The $\Delta \widetilde{E}$ estimates are computed by

$$
\Delta \widetilde{E}=\sum_{i=1}^{n} \Delta E\left(m_{i}, p_{i}\right),
$$

where $m_{i}$ is the mutation number $i, p_{i}$ is the residue position that the mutation $m_{i}$ should take effect on, $n$ is the total number of desired mutations and the dimension of the landscape, and $\Delta E$ is the result returned by (2) of mutation $m_{i}$ on position $p_{i}$. The estimates are calculated directly by summing values from the $\Delta E$ table. The estimates might deviate slightly from actual values since they do not take into account pairwise electrostatic and coulomb effects of the mutations with one another.

2.7. Predicting Amyloidogenicity. Although the stability results returned by the Htt-N-term landscape predict the effect of mutations on the native helical structure, the landscape does not reveal amyloidogenicity potentials of the mutations. Moreover, the 3-dimensional PDB conformation 
of the amyloid form of the Htt-N-term is unknown at the present time. Hence, it is difficult to estimate the energy barrier between native and amyloid Htt forms. Nevertheless, we resort to several state-of-the-art computational tools to approximate the 3-point mutations that are both stable and least amyloidogenic, which would result in slower amyloid formation and slower disease progression. In particular, we use Zyggregator [50], TANGO [51], and PASTA [52] to predict the aggregation propensities of the top ranking stable 3-point mutations. The top 3 mutations with best stability and lowest amyloidogenicity were run through an $\mathrm{MD}$ production to verify that they conserve the native helical structure of Htt-N-term.

\section{Results and Discussion}

To generate the mutation landscape of the Htt-N-term region of the huntingtin protein we computationally mutated each of the 17 amino acid positions in the $\mathrm{N}$-terminal region to each of the 20 canonical amino acids, as outlined by Algorithm 1. For each mutant structure, we calculated the total energy produced by introducing the respective mutation when computing the electrostatic, solvation, and enthalpy terms in (1). The complete mutation landscape is plotted in Figure 2. Mutations that increase the stability of the structure have the lowest energies, while mutations that destabilize the structure increase its energy. Table S1 (see Table S1 in Supplementary Material available online at http://dx.doi.org/10.1155/2016/6247867) ranks the top 100 mutations in decreasing order of stability. Our results outline that certain single-point mutations lead to an increase in the total energy of the Htt-N-term (mutations with red peaks), while others significantly stabilize the region, potentially lowering amyloidogenicity and aggregation rates of amyloids (mutations with purple nadirs) with respect to the first model of huntingtin aggregation outlined in Section 1 . This increase in stability is aimed at preventing self-association of the Htt$\mathrm{N}$-terminal region into helical bundles [9] and at lowering the potential of aggregation that is not mediated through an oligomeric precursor [53].

3.1. Increasing Htt-N-Terminal Stability to Prevent SelfAssociation and Lower Aggregation Propensity. Three specific mutations in the landscape of Figure 2 introduce a significant increase in structural energy, resulting in a less stable helical form. These three mutations indicate that the Htt-N-term sequence is most likely to destabilize when mutations are applied at the beginning of the sequence (position 1), in the middle (position 9), or at the end (position 17). These corresponding positions show energy spikes in the landscape that can possibly be attributed to the strain that the mutations introduce in the alpha-helical structure. When the Htt-Nterm is mutated at position 1 from a Methionine to Aspartic Acid (M1D) it causes an enormous increase in overall energy of the structure. Similarly, mutating the positively charged Lysine at position 9 to a hydrophobic polar Tyrosine (K9Y) and a hydrophobic Phenylalanine to a hydrophobic polar Tyrosine (F17Y) destabilizes the region. In turn, this destabilization could make it easier for the region to break away from its helical form. By decreasing the stability of the Htt-N-term dramatically, the process of aggregation may be increased significantly. Similar to other amyloid proteins in various diseases, the oligomerization of the monomeric forms of the Htt protein is initiated and promoted by the instability in the alpha-helical Htt-N-term region [14, 29, 30, 54]. Apart from the three mutations we mentioned above, the following mutations can also contribute in destabilizing it: L4P, L4M, K9F, K9M, F11T, E12D, S13Y, and S16R. If these mutations find their way into the Htt-N-terminal region, they can potentially promote beta strands to form into long beta-sheets $[14,32$, 49].

There are several reasons why certain mutations cause increases in the total energy of the sequence, which include effects caused by structural, electrostatic, and functional groups. Increasing the negative charge of the sequence by phosphorylation of the Htt-N-term region has been shown to decrease fibril formation and disease toxicity $[55,56]$. The destabilizing mutation at position 1 increases the total negative charge of the sequence. In conjunction with the electrostatic characteristics of aspartic acid, its steric properties could also contribute to the impairment of the alpha helix assembly which might promote the nucleation of beta-sheets into fibrils. Similarly, the mutations K9Y and F17Y could also have a similar outcome. Tyrosine's high polarity and steric hindrance as a result of its phenol group would make the assembly of the alpha helix extremely difficult. As a result, the rate of fibrillation of Htt proteins could drastically increase leading to higher toxicity in the body.

More importantly, the landscape suggests that various single-point mutations can stabilize the helical N-terminal region. More specifically, Figure 2(b) suggests that four types of mutations can introduce the greatest stability. First, introducing a Lysine $(\mathrm{K})$ into the helical structure can strengthen the bonds of the N-terminal region. This can be seen by the blue dots across the landscape that result from performing a mutation into a K. Similarly, introducing an Arginine (R) into the helical structure also appears to increase stability, regardless of which position it is introduced at (except at position 16). Third, most mutations at positions 15 and 17 result in blue dots, which translates to a relaxation of the structure and increased stability. A complete list of the effect of these mutations on structure energy is ordered in Table S1.

Since our first goal is to explore how to improve the stability of the Htt-N-terminal region to considerably lower amyloidogenicity and aggregation propensities, we can benefit more from mutations by considering the effect of simultaneous multiple-point mutations on the region's stability. We have recently shown that efficiently estimating the structural energy, $\Delta \widetilde{E}$, of multiple $n$-point mutations by summing the total effect of the $n$-single-point mutations separately returns accurate estimates so long as the mutations are not all adjacent [41]. We list the top 2-point and 3-point mutations of the Htt-N-terminal region in Tables S2 and S3, respectively, and include in Algorithm 2 the procedure we followed to generate these two lists. Moreover, we capture the best 20 mutation results in Table 1.

Although we can differentiate between the results in Table 1 according to stability, it is unclear which of the top 
TABle 1: The top 3-point mutations in Htt-N-term with lowest $\Delta \widetilde{E}$ values.

\begin{tabular}{|c|c|c|c|}
\hline Mutant & Sequence & $\Delta \widetilde{E}$ & Mutations \\
\hline M1 & ---KK---------D-- & -668.3 & L4K E5K K15D \\
\hline M2 & $--\mathrm{KK}----------\mathrm{D}--$ & -667.7 & T3K L4K K15D \\
\hline M3 & $--\mathrm{K}-\mathrm{K}---------\mathrm{D}--$ & -664.9 & T3K E5K K15D \\
\hline M4 & $---\mathrm{KK}---------\mathrm{E}--$ & -649 & L4K E5K K15E \\
\hline M5 & $------E--$ & -648.4 & T3K L4K K15E \\
\hline M6 & $---\mathrm{K}-------\mathrm{K}--\mathrm{D}--$ & -646.7 & L4K E12K K15D \\
\hline M7 & $--\mathrm{K}-\mathrm{K}---------\mathrm{E}--$ & -645.7 & T3K E5K K15E \\
\hline M8 & --KKK------------ & -645.6 & T3K L4K E5K \\
\hline M9 & $----\mathrm{K}-------\mathrm{K}--\mathrm{D}--$ & -644 & E5K E12K K15D \\
\hline M10 & $--\mathrm{K}--------\mathrm{K}--\mathrm{D}--$ & -643.4 & T3K E12K K15D \\
\hline M11 & $---\mathrm{K}----------\mathrm{D}-\mathrm{D}$ & -637.3 & L4K K15D F17D \\
\hline M12 & $----\mathrm{K}---------\mathrm{D}-\mathrm{D}$ & -634.5 & E5K K15D F17D \\
\hline M13 & $--\mathrm{K}-----------\mathrm{D}-\mathrm{D}$ & -633.9 & T3K K15D F17D \\
\hline M14 & $---\mathrm{K}----------\mathrm{D}-\mathrm{E}$ & -629.4 & L4K K15D F17E \\
\hline M15 & $-\mathrm{R}-\mathrm{K}-----------\mathrm{D}--$ & -629.2 & A2R L4K K15D \\
\hline M16 & $---\mathrm{K}-------\mathrm{K}--\mathrm{E}--$ & -627.5 & L4K E12K K15E \\
\hline M17 & $----\mathrm{K}---------\mathrm{D}-\mathrm{E}$ & -626.6 & E5K K15D F17E \\
\hline M18 & $-\mathrm{R}--\mathrm{K}----------\mathrm{D}--$ & -626.5 & A2R E5K K15D \\
\hline M19 & $--\mathrm{K}----------\mathrm{D}-\mathrm{E}$ & -626 & T3K K15D F17E \\
\hline M20 & $-\mathrm{RK}------------\mathrm{D}--$ & -625.8 & A2R T3K K15D \\
\hline
\end{tabular}

3-point mutations is the least amyloidogenic. The $\Delta \widetilde{E}$ values do not estimate the size of the energy barrier between the native and disordered states of the N-term region. Such an estimate would clearly indicate the effect of the mutations on amyloidogenicity and aggregation propensity. However, this can still be roughly estimated by existing tools. More precisely, we resorted to three tools, Zyggregator [50], TANGO [51], and PASTA [52], to predict the aggregation propensities of the top 3-point mutations. The results of each of the tools are listed in Tables 2-4. The results returned by Zyggregator in Table 2 do not suggest a difference in amyloidogenicity in the 20 sequences. The beta-sheet propensities are similar, the alpha-helical propensities only differ slightly, and the intrinsic aggregation propensities are within close range. The results returned by TANGO in Table 3 suggest that result M16 (L4K E12K K15E) has the lowest amyloidogenic potential, a relatively high helical potential, and one of the lowest betasheet propensities. The results returned by PASTA in Table 4 favor result M7 (T3K E5K K15E). Result M7 has the highest percentage of residues predicted to be in a helix state and is the most stable out of the set, resulting in the lowest aggregation potential. The stability results in Table 1 satisfy conditions from the first model of aggregation, suggesting that M16 and M7 are potentially the most stable Htt-Nterminal mutant forms.

3.2. Weakening Interactions between the Htt-N-Terminal Region and the PolyQ Domain. The second step of this multiobjective approach is to analyze which of the mutation combinations has likely weaken interactions with the polyQ domain, satisfying the condition set by the second model of aggregation (see Section 1). For this procedure, we considered mutations that replace hydrophobic residues on the Htt-Nterm with charged ones. In the domain cross-talk model, the hydrophobic residues are responsible for playing a part in stabilizing the polyQ domain. Altering hydrophobic residues to charged residues adds strain to the $\mathrm{Htt}-\mathrm{N}$-term and affects the stability and length of the polyQ domain, altering aggregation. From Table 1, we find that most mutation combinations in our list already substitute a hydrophobic amino acid with a charged reside (M1, M2, M4, M5, M6, M8, M11, M14, M15, M16, M18, and M20). Although the Htt$\mathrm{N}$-terminal region contains several hydrophobic residues, it has been shown that removing just one of the hydrophobic amino acids can effectively weaken the interactions with the polyQ domain and potentially reduce overall aggregation [9]. We acknowledge that we cannot quantitatively model the interactions of the mutations with the polyQ domain without a good PDB representation; however, we are relying on the theoretical understanding behind the interactions of the hydrophobic residues and the polyQ domain to conclude that the charged residues with weaken interactions.

3.3. Breaking the Solvent-Inaccessible Intermolecular Side Chain of the Htt-N-Terminal Region. In addition to satisfying the first two conditions of helical stability and hydrophilic substitution, the M16 combination luckily also contains a K15E substitution. Substituting the Lysine residue at position 15 breaks the solvent-inaccessible intermolecular side chain interaction that contributes to oligomerization [9].

Since we are introducing three mutations to the Htt$\mathrm{N}$-terminal structure and lowering the stability by a large factor, it remains to check that the mutations do not alter the $3 \mathrm{D}$ helical conformation of the region. To check this, we performed a complete molecular dynamics simulation of 50 nanoseconds on the most stable mutants from Table 1 (M1, M7, and M16) and found that indeed the Htt-N-terminal region maintains its stability in all three structures. We report the results of the RMSD and RMSF graphs of each mutant structure in Figure 3. Figures 3(a), 3(c), and 3(e) plot the RMSD fluctuations over time and Figures 3(b), 3(d), and 3(f) plot the RMSF graphs indicating the stability of the mutated residue positions during the respective MD run. The RMSF graphs show that the regions around the mutated residues are relatively stable and the RMSD graphs all report values less than $1 \mathrm{~nm}$. While all the three mutant structures appear to exhibit good stability, we observe that mutant M16 shows less RMSD variations than M1 and more stable RMSF values than M7 at the mutated residues (positions 4, 5, and 15). For all the reasons we explored above, we believe that the M16 mutant (L4K E12K K15E) is the best mutation candidate to lower the amyloidogenicity of $\mathrm{Htt}$ in both models of huntingtin aggregation.

\section{Conclusion}

The Htt protein is a huge protein that contains 3144 amino acids. One limitation of the study is that we did not consider the entire structure in our energy predictions. The simulation 
TABLE 2: Aggregation potentials of top 20 results for Htt-N-term 3-point mutations computed by Zyggregator.

\begin{tabular}{|c|c|c|c|c|c|}
\hline Mutant & Hydrophobicity & Charge & Beta-sheet propensity & Alpha-helical propensity & Aggregation propensity \\
\hline Native & 4.91 & 1 & 74.04 & 75.00 & -5.01 \\
\hline M1 & 10.40 & 2 & 73.87 & 74.39 & -5.37 \\
\hline M2 & 12.31 & 1 & 73.71 & 75.01 & -5.37 \\
\hline M3 & 7.58 & 2 & 73.87 & 75.04 & -5.49 \\
\hline M4 & 9.50 & 2 & 73.87 & 74.87 & -5.50 \\
\hline M5 & 11.41 & 1 & 73.71 & 75.49 & -5.50 \\
\hline M6 & 10.40 & 2 & 73.87 & 74.39 & -5.37 \\
\hline M7 & 6.68 & 2 & 73.87 & 75.52 & -5.62 \\
\hline M8 & 11.13 & 5 & 73.71 & 75.30 & -6.70 \\
\hline M9 & 5.67 & 3 & 74.04 & 74.42 & -5.48 \\
\hline M10 & 7.58 & 2 & 73.87 & 75.04 & -5.49 \\
\hline M11 & 16.62 & -1 & 73.58 & 74.24 & -5.30 \\
\hline M12 & 11.89 & 0 & 73.74 & 74.27 & -4.78 \\
\hline M13 & 13.80 & -1 & 73.58 & 74.89 & -5.42 \\
\hline M14 & 15.72 & -1 & 73.58 & 74.72 & -5.43 \\
\hline M15 & 14.88 & 1 & 74.10 & 74.67 & -5.24 \\
\hline M16 & 9.50 & 2 & 73.87 & 74.87 & -5.50 \\
\hline M17 & 10.99 & 0 & 73.74 & 74.75 & -4.91 \\
\hline M18 & 10.15 & 2 & 74.26 & 74.70 & -5.35 \\
\hline M19 & 12.90 & -1 & 73.58 & 75.37 & -5.55 \\
\hline M20 & 12.06 & 1 & 74.10 & 75.33 & -5.36 \\
\hline
\end{tabular}

TABLE 3: Aggregation potentials of top 20 results for Htt-N-term 3point mutations computed by TANGO.

\begin{tabular}{|c|c|c|c|c|}
\hline Mutant & Amylo & Turn & Helix & Beta \\
\hline Native & 0.55 & 3.17 & 28.74 & 15.36 \\
\hline M1 & 4.18 & 6.31 & 1.26 & 20.41 \\
\hline M2 & 0.57 & 6.38 & 0.00 & 18.00 \\
\hline M3 & 0.57 & 6.16 & 0.00 & 20.56 \\
\hline M4 & 4.21 & 3.67 & 1.26 & 15.58 \\
\hline M5 & 0.57 & 3.75 & 0.00 & 13.17 \\
\hline M6 & 0.00 & 6.11 & 16.82 & 19.46 \\
\hline M7 & 0.57 & 3.53 & 0.00 & 15.73 \\
\hline M8 & 0.57 & 3.64 & 0.00 & 11.63 \\
\hline M9 & 0.00 & 5.87 & 9.52 & 26.75 \\
\hline M10 & 0.00 & 5.95 & 8.95 & 21.72 \\
\hline M11 & 0.56 & 5.92 & 7.97 & 19.19 \\
\hline M12 & 4.16 & 5.65 & 6.32 & 26.40 \\
\hline M13 & 0.56 & 5.75 & 1.85 & 21.45 \\
\hline M14 & 0.56 & 5.80 & 7.97 & 19.42 \\
\hline M15 & 0.08 & 6.54 & 0.00 & 20.13 \\
\hline M16 & 0.00 & 3.49 & 17.05 & 14.66 \\
\hline M17 & 4.16 & 5.53 & 6.32 & 26.63 \\
\hline M18 & 0.95 & 6.24 & 0.00 & 27.17 \\
\hline M19 & 0.57 & 5.63 & 1.85 & 21.68 \\
\hline M20 & 0.34 & 6.25 & 1.06 & 23.01 \\
\hline
\end{tabular}

The Amylo column of the results returned by TANGO suggests that mutant M16 has the least amyloidogenicity.
TABLE 4: Aggregation potentials of top 20 results for Htt-N-term 3point mutations computed by PASTA.

\begin{tabular}{lccc}
\hline Mutant & Best energy & $\% \alpha$-helix & $\%$ coil \\
\hline Native & -1.12 & 76.47 & 23.53 \\
M1 & -0.48 & 70.59 & 29.41 \\
M2 & -0.48 & 76.47 & 23.53 \\
M3 & -0.90 & 76.47 & 23.53 \\
M4 & -0.82 & 70.59 & 29.41 \\
M5 & -0.82 & 76.47 & 23.53 \\
M6 & -1.07 & 70.59 & 29.41 \\
M7 & -1.50 & 82.35 & 17.65 \\
M8 & -1.00 & 76.47 & 23.53 \\
M9 & -0.68 & 76.47 & 23.53 \\
M10 & -1.12 & 76.47 & 23.53 \\
M11 & -1.07 & 64.71 & 35.29 \\
M12 & -0.90 & 76.47 & 23.53 \\
M13 & -1.12 & 76.47 & 23.53 \\
M14 & -1.07 & 64.71 & 35.29 \\
M15 & -0.91 & 70.59 & 29.41 \\
M16 & -1.07 & 70.59 & 29.41 \\
M17 & -0.90 & 76.47 & 23.53 \\
M18 & -0.90 & 76.47 & 23.53 \\
M19 & -1.12 & 76.47 & 23.53 \\
M20 & -1.12 & 76.47 & 23.53 \\
\hline
\end{tabular}

Mutant M7 results in the lowest energy, highest $\% \alpha$-helix, and lowest $\%$ coil. 


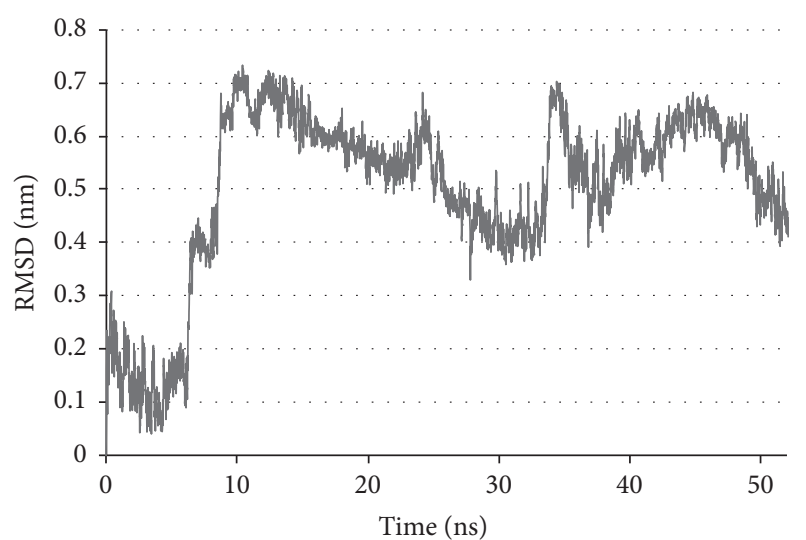

(a) RMSD graph for the Htt-N-term M1 mutant

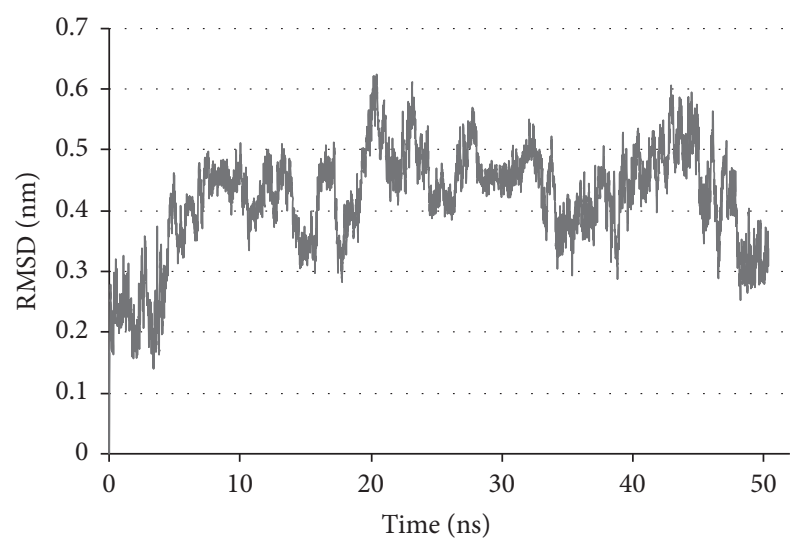

(c) RMSD graph for the Htt-N-term M7 mutant

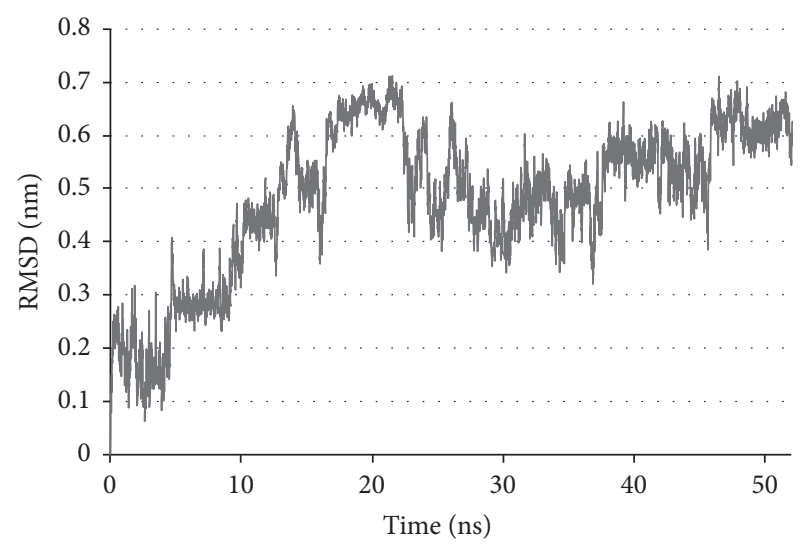

(e) RMSD graph for the Htt-N-term M16 mutant

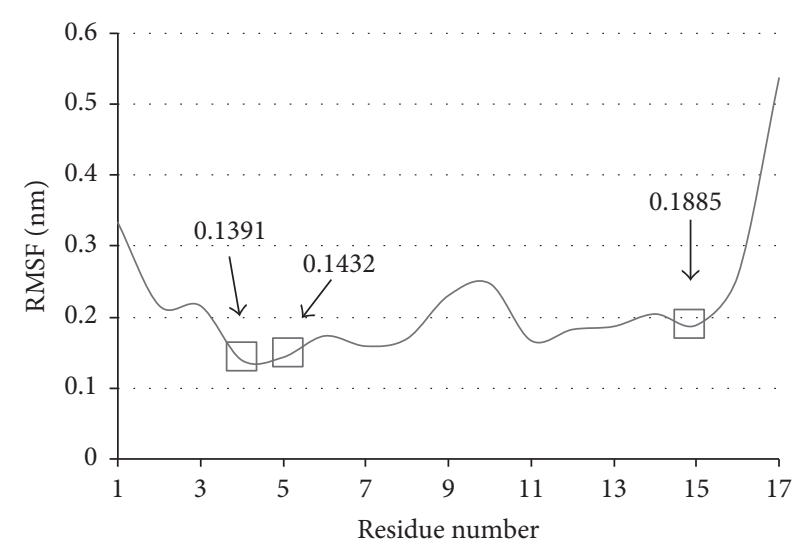

(b) RMSF graph for the Htt-N-term M1 mutant

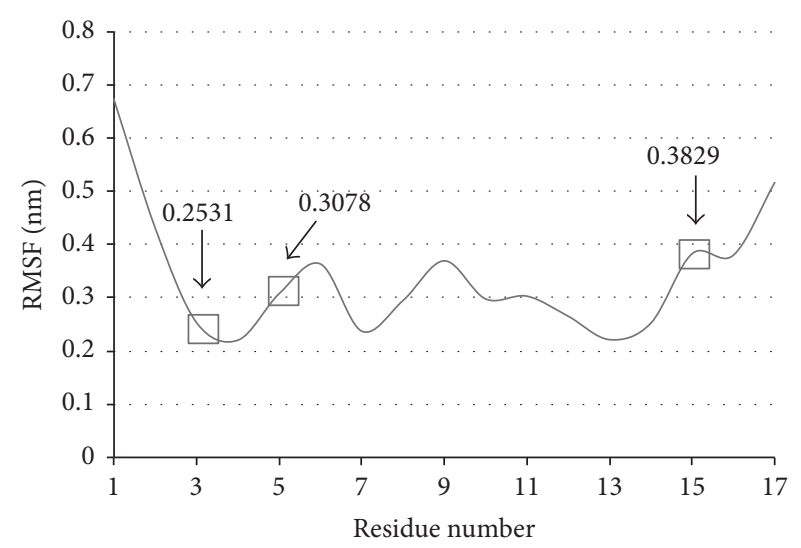

(d) RMSF graph for the Htt-N-term M7 mutant

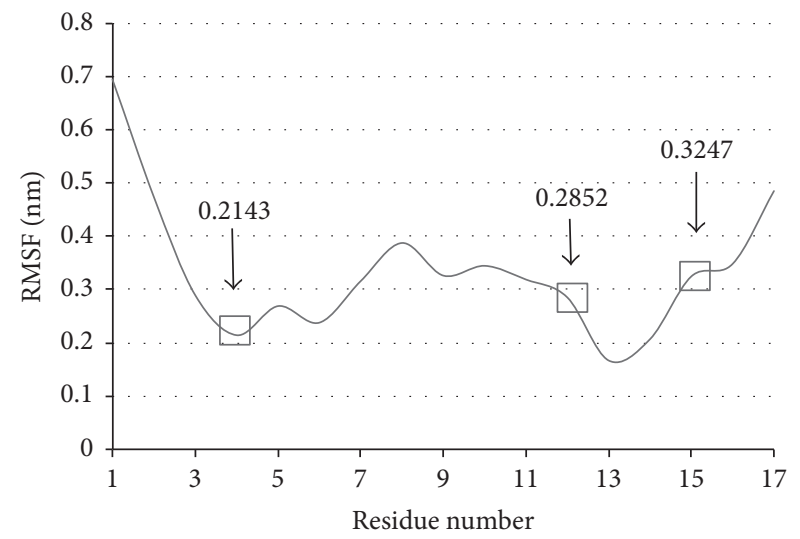

(f) RMSF graph for the Htt-N-term M16 mutant

FIgURE 3: MD results of mutants 1, 7, and 16. (a) A 52 ns RMSD plot of mutant (L4K E5K K15D) and (b) the RMSF plot of the same mutant. (c) A 50 ns RMSD plot of mutant (T3K E5K K15E) and (d) the RMSF values of the same mutant. (e) a 52 ns RMSD plot of mutant (L4K E12K $\mathrm{K} 15 \mathrm{E}$ ) and (f) the RMSF values of the same mutant. All structures appear to be stable and conserved.

run-time would have been excessive and it was therefore not possible to conduct MD simulations and stability analysis on the entire protein. However, many studies have consistently reported valid findings by only examining the $\mathrm{N}$-term region of $\mathrm{Htt}$ and considered it the most important component to study in the progression of Huntington's disease [11-14, 47, 57].
The two models of huntingtin aggregation describe the role of the Htt-N-terminal region in (1) utilizing alphahelical interactions to create oligomers and (2) the role of its hydrophobic residues in stabilizing the polyQ domain and exciting aggregation. We explored the mutation landscape of this structure by mutating amino acid residues and calculating the resulting changes in total energy. Our goal 
was to probe the entire mutation landscape of the Htt-Nterminal region to identify mutations that would lower the aggregation propensities outlined in both Htt models. By identifying critical maxima and minima points in the landscape, experimentalists have a smaller subset of possibilities to test for mutants that change HD progression rates.

Exploring the entire stability landscape of the Htt-Nterminal region enabled us to identify mutation combinations that significantly increase helical stability to prevent selfassociation of the Htt-N-terminal region into helical bundles and lower the aggregation propensity suggested by the first model (see Section 1 for model descriptions). We investigated the mutation landscape of the Htt-N-term to unravel unstable regions characterized by high energy values while identifying regions that can further stabilize the Htt-N-term. By narrowing our search space for lowest amyloidogenic potential mutations, we calculated the $\Delta \widetilde{E}$ for multiple-point mutations (2-point and 3-point). We focused on mutants that possessed the lowest energies and used state-of-the-art tools to predict which of those mutations have promising low amyloidogenic propensities. We analyzed the most promising mutation combinations that additionally replace hydrophobic residues with charged amino acids to weaken interactions with the polyQ domain to lower the aggregation resulting from interaction with the polyQ domain. Out of the millions of 3-point mutations that we considered, the (L4K E12K K15E) mutant exhibited extreme stability, low amyloidogenicity, hydrophobic replacement, and removal of the solvent-inaccessible intermolecular side chain that assists oligomerization. The results we explored computationally can serve as possibilities that experimentalists can potentially utilize.

\section{Competing Interests}

The authors declare that they have no competing interests.

\section{Authors' Contributions}

Mohamed R. Smaoui and Jérôme Waldispühl designed the study. Mohamed R. Smaoui and Cody Mazza-Anthony developed code, carried out the simulations, and drafted the manuscript. Mohamed R. Smaoui, Cody Mazza-Anthony, and Jérôme Waldispühl analyzed results and approved the final manuscript.

\section{Acknowledgments}

Mohamed R. Smaoui was supported by a postdoctoral fellowship from the Fonds de Recherche Nature et Technologies Quebec. Jérôme Waldispühl was supported by a Discovery grant from the Natural Science and Engineering Research Council of Canada. MD productions were facilitated by the Calcul Quebec and Compute Canada Networks and the computing facilities at the McGill High Performance Computing Center.

\section{References}

[1] M. Macdonald, "A novel gene containing a trinucleotide repeat that is expanded and unstable on Huntington's disease chromosomes," Cell, vol. 72, no. 6, pp. 971-983, 1993.
[2] C. A. Ross and S. J. Tabrizi, "Huntington's disease: from molecular pathogenesis to clinical treatment," The Lancet Neurology, vol. 10, no. 1, pp. 83-98, 2011.

[3] P.T. J. Lansbury, "Evolution of amyloid: what normal protein folding may tell us about fibrillogenesis and disease," Proceedings of the National Academy of Sciences of the United States of America, vol. 96, no. 7, pp. 3342-3344, 1999.

[4] C. M. Dobson, "Protein misfolding, evolution and disease," Trends in Biochemical Sciences, vol. 24, no. 9, pp. 329-332, 1999.

[5] F. Chiti and C. M. Dobson, "Protein misfolding, functional amyloid, and human disease," Annual Review of Biochemistry, vol. 75, pp. 333-366, 2006.

[6] C. A. Ross and M. A. Poirier, "Protein aggregation and neurodegenerative disease," Nature Medicine, vol. 10, pp. S10-S17, 2004.

[7] M. Ramirez-Alvarado, J. S. Merkel, and L. Regan, "A systematic exploration of the influence of the protein stability on amyloid fibril formation in vitro," Proceedings of the National Academy of Sciences, vol. 97, no. 16, pp. 8979-8984, 2000.

[8] C. L. Hoop, H.-K. Lin, K. Kar et al., "Polyglutamine amyloid core boundaries and flanking domain dynamics in huntingtin fragment fibrils determined by solid-state nuclear magnetic resonance," Biochemistry, vol. 53, no. 42, pp. 6653-6666, 2014.

[9] J. R. Arndt, S. G. Kondalaji, M. M. Maurer, A. Parker, J. Legleiter, and S. J. Valentine, "Huntingtin N-terminal monomeric and multimeric structures destabilized by covalent modification of heteroatomic residues," Biochemistry, vol. 54, no. 28, pp. 42854296, 2015

[10] A. Bhattacharyya, A. K. Thakur, V. M. Chellgren et al., "Oligoproline effects on polyglutamine conformation and aggregation," Journal of Molecular Biology, vol. 355, no. 3, pp. 524-535, 2006.

[11] G. Darnell, J. P. R. O. Orgel, R. Pahl, and S. C. Meredith, "Flanking polyproline sequences inhibit $\beta$-sheet structure in polyglutamine segments by inducing PPII-like helix structure," Journal of Molecular Biology, vol. 374, no. 3, pp. 688-704, 2007.

[12] E. Rockabrand, N. Slepko, A. Pantalone et al., "The first 17 amino acids of Huntingtin modulate its sub-cellular localization, aggregation and effects on calcium homeostasis," Human Molecular Genetics, vol. 16, no. 1, pp. 61-77, 2007.

[13] G. D. Darnell, J. Derryberry, J. W. Kurutz, and S. C. Meredith, "Mechanism of cis-inhibition of polyQ fibrillation by polyP: PPII oligomers and the hydrophobic effect," Biophysical Journal, vol. 97, no. 8, pp. 2295-2305, 2009.

[14] A. K. Thakur, M. Jayaraman, R. Mishra et al., "Polyglutamine disruption of the huntingtin exon $1 \mathrm{~N}$ terminus triggers a complex aggregation mechanism," Nature Structural and Molecular Biology, vol. 16, no. 4, pp. 380-389, 2009.

[15] J. R. Arndt, R. J. Brown, K. A. Burke, J. Legleiter, and S. J. Valentine, "Lysine residues in the $\mathrm{N}$-terminal huntingtin amphipathic $\alpha$-helix play a key role in peptide aggregation," Journal of Mass Spectrometry, vol. 50, no. 1, pp. 117-126, 2015.

[16] H. Goehler, M. Lalowski, U. Stelzl et al., "A protein interaction network links GIT1, an enhancer of huntingtin aggregation, to Huntington's disease," Molecular Cell, vol. 15, no. 6, pp. 853-865, 2004.

[17] W.-C. M. Lee, M. Yoshihara, and J. T. Littleton, "Cytoplasmic aggregates trap polyglutamine-containing proteins and block axonal transport in a Drosophila model of Huntington's disease," Proceedings of the National Academy of Sciences of the United States of America, vol. 101, no. 9, pp. 3224-3229, 2004. 
[18] A. Pal, F. Severin, B. Lommer, A. Shevchenko, and M. Zerial, "Huntingtin-HAP40 complex is a novel Rab5 effector that regulates early endosome motility and is up-regulated in Huntington's disease," Journal of Cell Biology, vol. 172, no. 4, pp. 605618,2006

[19] M. DiFiglia, E. Sapp, K. Chase et al., "Huntingtin is a cytoplasmic protein associated with vesicles in human and rat brain neurons," Neuron, vol. 14, no. 5, pp. 1075-1081, 1995.

[20] D. Housman, "Gain of glutamines, gain of function?" Nature Genetics, vol. 10, no. 1, pp. 3-4, 1995.

[21] B. R. Leavitt, J. A. Guttman, J. G. Hodgson et al., "Wild-type huntingtin reduces the cellular toxicity of mutant huntingtin in vivo," American Journal of Human Genetics, vol. 68, no. 2, pp. 313-324, 2001.

[22] B. R. Leavitt, C. L. Wellington, and M. R. Hayden, "Recent insights into the molecular pathogenesis of Huntington disease," Seminars in Neurology, vol. 19, no. 4, pp. 385-395, 1999.

[23] F. O. Walker, "Huntington's disease: the road to progress," The Lancet Neurology, vol. 12, no. 7, pp. 624-625, 2013.

[24] J. S. Paulsen, R. E. Ready, J. M. Hamilton, M. S. Mega, and J. L. Cummings, "Neuropsychiatric aspects of Huntington's disease," Journal of Neurology Neurosurgery and Psychiatry, vol. 71, no. 3, pp. 310-314, 2001.

[25] L. R. Gauthier, B. C. Charrin, M. Borrell-Pagès et al., "Huntingtin controls neurotrophic support and survival of neurons by enhancing BDNF vesicular transport along microtubules," Cell, vol. 118, no. 1, pp. 127-138, 2004.

[26] C. Zuccato and E. Cattaneo, "Role of brain-derived neurotrophic factor in Huntington's disease," Progress in Neurobiology, vol. 81, no. 5-6, pp. 294-330, 2007.

[27] K. Hammond and B. Tatum, The Behavioral Symptoms of Huntington's Disease, Huntington's Outreach Project for Education, Stanford, Calif, USA, 2010.

[28] C. D. Clelland, R. A. Barker, and C. Watts, "Cell therapy in Huntington disease," Neurosurgical Focus, vol. 24, no. 3-4, article no. E8, 2008.

[29] M. Jayaraman, R. Kodali, B. Sahoo et al., "Slow amyloid nucleation via $\alpha$-helix-rich oligomeric intermediates in short polyglutamine-containing huntingtin fragments," Journal of Molecular Biology, vol. 415, no. 5, pp. 881-899, 2012.

[30] V. N. Sivanandam, M. Jayaraman, C. L. Hoop, R. Kodali, R. Wetzel, and P. C. A. Van Der Wel, "The aggregation-enhancing huntingtin N-terminus is helical in amyloid fibrils," Journal of the American Chemical Society, vol. 133, no. 12, pp. 4558-4566, 2011.

[31] N. W. Kelley, X. Huang, S. Tam, C. Spiess, J. Frydman, and V. S. Pande, "The predicted structure of the headpiece of the huntingtin protein and its implications on huntingtin aggregation," Journal of Molecular Biology, vol. 388, no. 5, pp. 919-927, 2009.

[32] S. Tam, C. Spiess, W. Auyeung et al., "The chaperonin TRiC blocks a huntingtin sequence element that promotes the conformational switch to aggregation," Nature Structural and Molecular Biology, vol. 16, no. 12, pp. 1279-1285, 2009.

[33] T. E. Williamson, A. Vitalis, S. L. Crick, and R. V. Pappu, "Modulation of polyglutamine conformations and dimer formation by the N-terminus of huntingtin," Journal of Molecular Biology, vol. 396, no. 5, pp. 1295-1309, 2010.

[34] S. L. Crick, K. M. Ruff, K. Garai, C. Frieden, and R. V. Pappu, "Unmasking the roles of $\mathrm{N}$ - and $\mathrm{C}$-terminal flanking sequences from exon 1 of huntingtin as modulators of polyglutamine aggregation," Proceedings of the National Academy of Sciences of the United States of America, vol. 110, no. 50, pp. 20075-20080, 2013.

[35] S. B. Needleman and C. D. Wunsch, "A general method applicable to the search for similarities in the amino acid sequence of two proteins," Journal of Molecular Biology, vol. 48, no. 3, pp. 443-453, 1970.

[36] R. V. Eck and M. O. Dayhoff, "Evolution of the structure of ferredoxin based on living relics of primitive amino acid sequences," Science, vol. 152, no. 3720, pp. 363-366, 1966.

[37] L. Pauling, E. Zuckerkandl, T. Henriksen, and R. Lövstad, "Chemical paleogenetics. Molecular 'restoration studies' of extinct forms of life," Acta Chemica Scandinavica, vol. 17, pp. 916, 1963.

[38] M. Levitt and A. Warshel, "Computer simulation of protein folding," Nature, vol. 253, no. 5494, pp. 694-698, 1975.

[39] E. E. Schadt, M. D. Linderman, J. Sorenson, L. Lee, and G. P. Nolan, "Computational solutions to large-scale data management and analysis," Nature Reviews Genetics, vol. 11, no. 9, pp. 647-657, 2010.

[40] D. Noble, “The rise of computational biology," Nature Reviews Molecular Cell Biology, vol. 3, no. 6, pp. 459-463, 2002.

[41] M. R. Smaoui and J. Waldispühl, "Complete characterization of the mutation landscape reveals the effect on amylin stability and amyloidogenicity," Proteins: Structure, Function and Bioinformatics, vol. 83, no. 6, pp. 1014-1026, 2015.

[42] S. Pronk, S. Páll, R. Schulz et al., "GROMACS 4.5: a highthroughput and highly parallel open source molecular simulation toolkit," Bioinformatics, vol. 29, no. 7, pp. 845-854, 2013.

[43] C. Oostenbrink, A. Villa, A. E. Mark, and W. F. van Gunsteren, "A biomolecular force field based on the free enthalpy of hydration and solvation: the GROMOS force-field parameter sets 53A5 and 53A6," Journal of Computational Chemistry, vol. 25, no. 13, pp. 1656-1676, 2004.

[44] H. J. C. Berendsen, J. P. M. Postma, W. F. van Gunsteren, and J. Hermans, "Interaction models for water in relation to protein hydration," in Intermolecular Forces, 1981.

[45] T. Darden, D. York, and L. Pedersen, "Particle mesh Ewald: an $N \cdot \log (N)$ method for Ewald sums in large systems," The Journal of Chemical Physics, vol. 98, no. 12, pp. 10089-10092, 1993.

[46] U. Essmann, L. Perera, M. L. Berkowitz, T. Darden, H. Lee, and L. G. Pedersen, "A smooth particle mesh Ewald method," The Journal of Chemical Physics, vol. 103, no. 19, pp. 8577-8593, 1995.

[47] P. Koehl and M. Delarue, "AQUASOL: an efficient solver for the dipolar Poisson-Boltzmann-Langevin equation," The Journal of Chemical Physics, vol. 132, no. 6, Article ID 064101, 2010.

[48] C. Azuara, H. Orland, M. Bon, P. Koehl, and M. Delarue, "Incorporating dipolar solvents with variable density in poissonboltzmann electrostatics," Biophysical Journal, vol. 95, no. 12, pp. 5587-5605, 2008.

[49] G. G. Krivov, M. V. Shapovalov, and R. L. Dunbrack Jr., "Improved prediction of protein side-chain conformations with SCWRL4," Proteins: Structure, Function and Bioinformatics, vol. 77, no. 4, pp. 778-795, 2009.

[50] G. G. Tartaglia, A. P. Pawar, S. Campioni, C. M. Dobson, F. Chiti, and M. Vendruscolo, "Prediction of aggregation-prone regions in structured proteins," Journal of Molecular Biology, vol. 380, no. 2, pp. 425-436, 2008.

[51] A.-M. Fernandez-Escamilla, F. Rousseau, J. Schymkowitz, and L. Serrano, "Prediction of sequence-dependent and mutational effects on the aggregation of peptides and proteins," Nature Biotechnology, vol. 22, no. 10, pp. 1302-1306, 2004. 
[52] I. Walsh, F. Seno, S. C. E. Tosatto, and A. Trovato, "PASTA 2.0: an improved server for protein aggregation prediction," Nucleic Acids Research, vol. 42, no. 1, pp. W301-W307, 2014.

[53] C. T. Aiken, J. S. Steffan, C. M. Guerrero et al., "Phosphorylation of threonine 3: implications for huntingtin aggregation and neurotoxicity," Journal of Biological Chemistry, vol. 284, no. 43, pp. 29427-29436, 2009.

[54] R. Mishra, M. Jayaraman, B. P. Roland et al., "Inhibiting the nucleation of amyloid structure in a huntingtin fragment by targeting $\alpha$-helix-rich oligomeric intermediates," Journal of Molecular Biology, vol. 415, no. 5, pp. 900-917, 2012.

[55] R. Mishra, C. L. Hoop, R. Kodali, B. Sahoo, P. C. A. Van Der Wel, and R. Wetzel, "Serine phosphorylation suppresses huntingtin amyloid accumulation by altering protein aggregation properties," Journal of Molecular Biology, vol. 424, no. 1-2, pp. 1-14, 2012.

[56] X. Gu, E. R. Greiner, R. Mishra et al., "Serines 13 and 16 are critical determinants of full-length human mutant huntingtin induced disease pathogenesis in HD mice," Neuron, vol. 64, no. 6, pp. 828-840, 2009.

[57] B. Hess, C. Kutzner, D. Van Der Spoel, and E. Lindahl, "GROMACS 4: algorithms for highly efficient, load-balanced, and scalable molecular simulation," Journal of Chemical Theory and Computation, vol. 4, no. 3, pp. 435-447, 2008. 


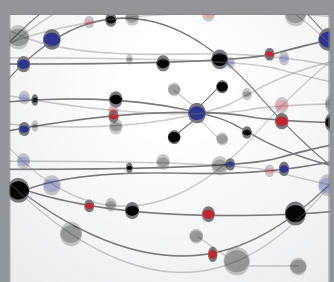

The Scientific World Journal
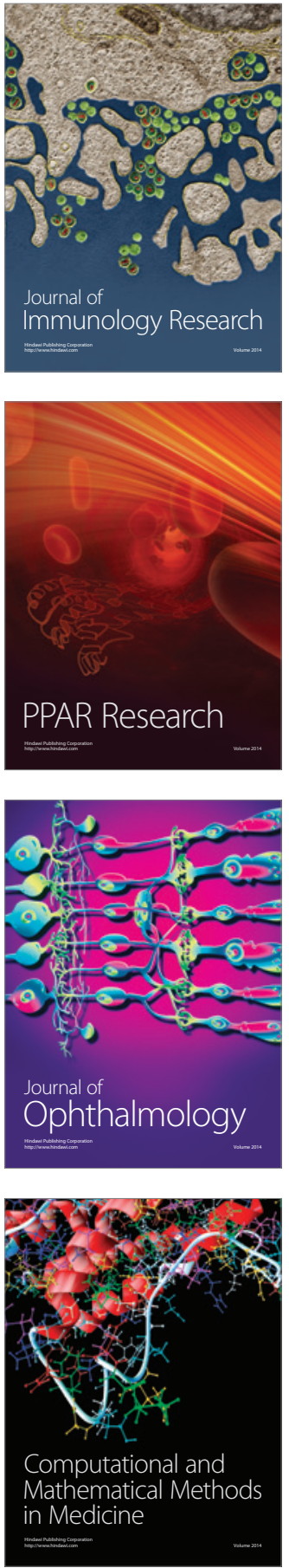

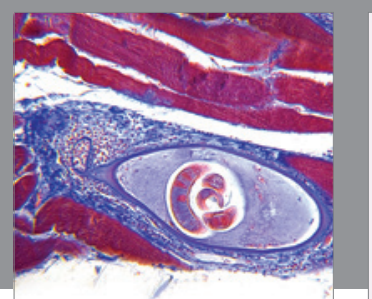

Gastroenterology Research and Practice

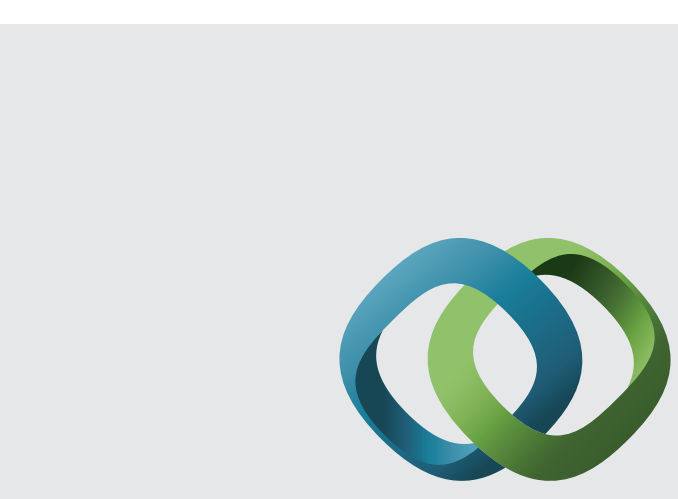

\section{Hindawi}

Submit your manuscripts at

http://www.hindawi.com
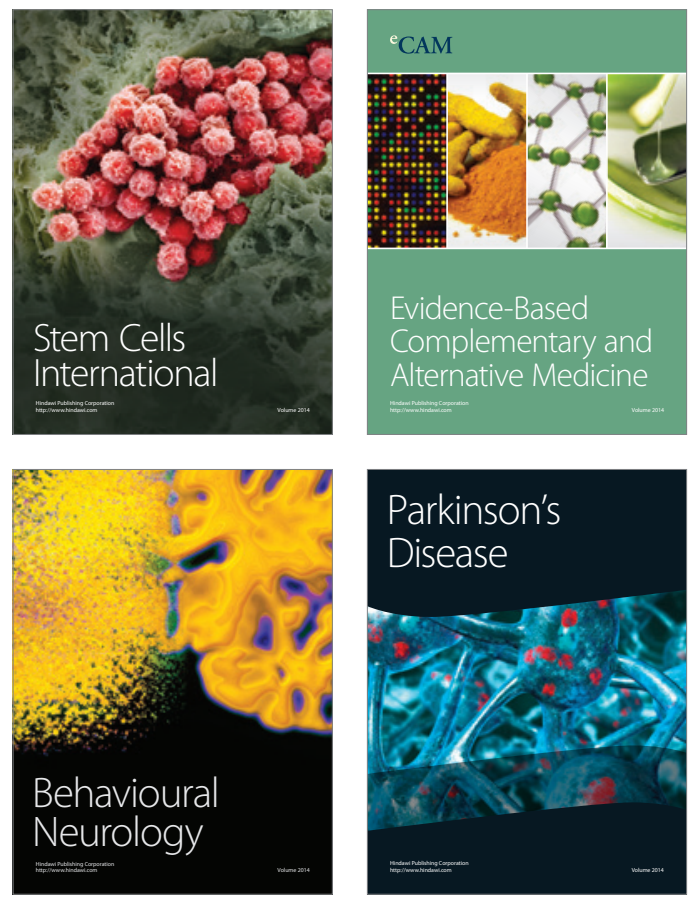
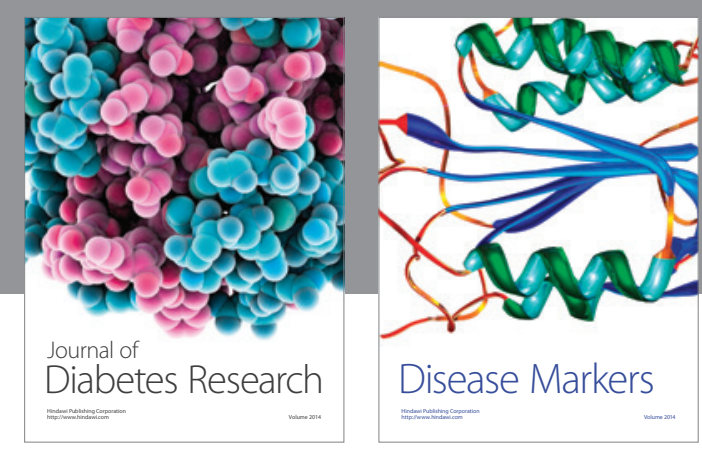

Disease Markers
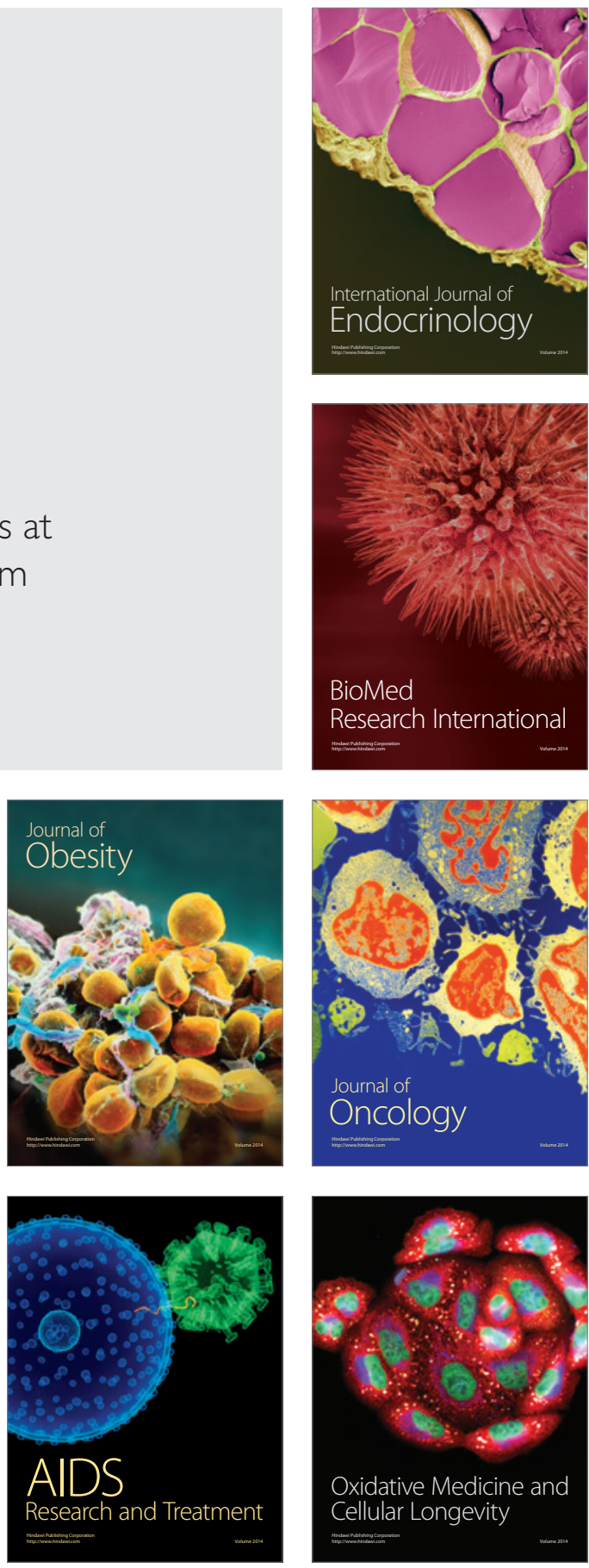